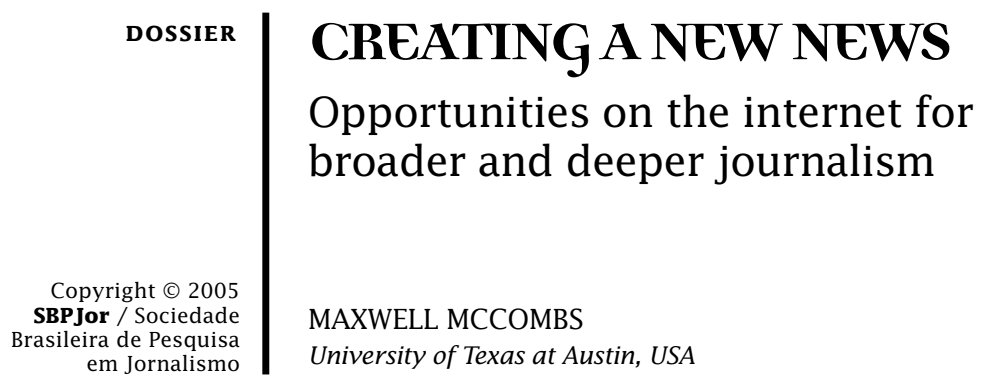

ABSTRACT Drawing upon two established ideas, the concept of the long tail from statistics and the concept of an agenda from agenda-setting theory, this issue will describe horizontal and vertical strategies that provide a platform for both planning and then evaluating through formal research broad programs of systematic experimentation with new forms of content. These new forms do not change the fundamental values of journalism, but they do offer an opportunity for creating an enhanced news package on the internet that is compelling journalism.

Key-Words: journalism, internet, long-tail, news, forms, content.

"You can have a Ford in any color, as long as it is black."

- Henry Ford

\title{
Introduction
}

HENRY Ford's SUCCINCT SUMmARY OF CONSUMER OPTIONS FOR AN AUTOMOBILE IN THE EARLY days of the $20^{\text {th }}$ century is a metaphoric description of our options for a daily news package across the past century. With minor exceptions, everyone in the community received the same "black Ford," a set of news articles and features that were a greatly truncated microcosm of what was happening out there in the world. Journalists rely upon a set of professional news values to winnow a small number of "newsworthy" events and trends from the vast events of the day. And due to the constraints of the news hole and broadcast time, even this small number is further reduced in the daily news report received by the public.

However, the internet has set the stage for a vastly expanded approach to news, a much broader and deeper version of the news than traditionally has been offered to the public in daily newspapers and television broadcasts. Although there are many distinctive new features of the internet and online journalism, such as a vastly increased level of interactivity between the public and journalists and 
the accessibility of internet news sources via an ever-growing host of small electronic devices, the success - some would argue, the survival - of quality journalism hinges more on content than on the attributes of any communication medium per se. The medium is only the distribution system. In MediaMorphosis: Understanding New Media, Roger Fidler noted, "People do not buy information technologies - they buy content, usefulness, and convenience at the point when they perceive value to match the cost." 1

Using the internet primarily as a distribution channel for an online newspaper that is more or less the same package of news as the traditional printed version will enjoy only limited success. The major factors behind the continuing decline in U.S. daily newspaper circulation, for example, are not the appeal of new technologies or any intrinsic dislike for a print medium. The serious decline in circulation predates the internet and all these new technologies by several decades because large numbers of the public simply have not found the content of newspapers sufficiently relevant and compelling. 2 Now the internet offers an opportunity to engage the interests and needs of the public with a much wider array of news. More specifically, a vastly expanded news package on the internet can increase the resonance of the newspaper for the individual interests of readers.

Although we think of a newspaper as a mass medium, the average news story is selected by only one reader in four. The newspaper audience is composed of many small sub-publics that select very different sets of news from the package that is printed and distributed. A key attribute of the new media landscape is the vast capacity of an internet site, a capacity that stands in stark contrast to the constraints of the limited news hole in daily newspapers or limited broadcast time for television. This immensely enlarged capacity of news packages housed on the internet offers the opportunity for news organizations to better serve the needs of these numerous sub-publics with a broader and deeper array of news stories and information.

Drawing upon two established ideas, the concept of the long tail from statistics 3 and the concept of an agenda from agenda-setting theory, 4 this presentation will describe horizontal and vertical strategies that provide a platform for both planning and then evaluating through formal research broad programs of systematic experimentation with new forms of content. These new forms do not change the fundamental values of journalism, but they do offer an opportunity for creating an enhanced news package on the internet that is compelling journalism. 
Taking advantage of the internet's long tail, a horizontal strategy for this new news can extend its reach to include a greatly expanded set of stories, listings and background information. Taking further advantage of the internet's long tail and incorporating the concept of an attribute agenda, a vertical strategy for the new news also can increase the depth of its coverage by offering a wider range of perspectives on the major events and issues of the day.

\section{Horizontal strategy}

The concept of the long tail is a useful model for expanding the breadth and relevance of our news packages to the public. Originally a statistical concept, the long tail describes an oligopoly, a set of units - such as book titles, musical recordings, news stories, or whatever - in which a few units account for a large proportion of the total use by the public, but in which a very, very large number of unique units account for the remainder of the use. These kinds of situations that are described by a large, compressed head and long tail are found in many settings. In The Long Tail, Chris Anderson applied this idea to a variety of commercial internet settings. Compared to traditional retailers who must emphasize the head in determining their inventory of items for sale, we see in Table 1 that e-commerce retailers generate a significant portion of their sales from their long tail inventory. The public is interested in much more than just "hits" and "bestsellers."

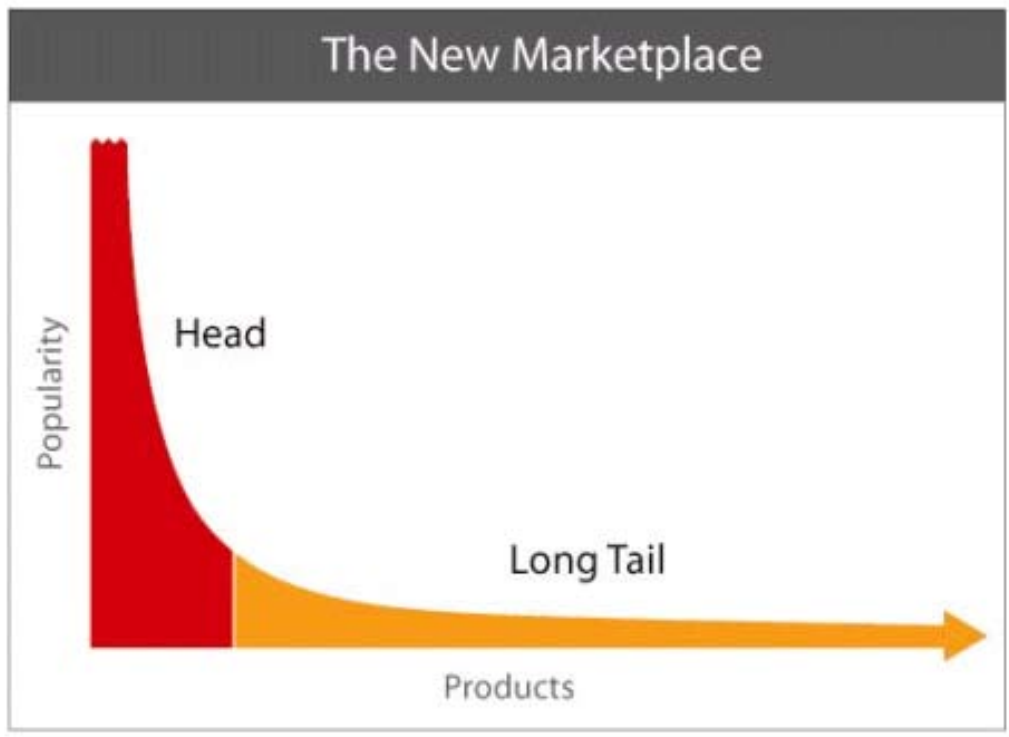


TABLE 1. Heads and Tails in the Sales of Cultural Products: Comparisons of Retailing Based on The Head With Retailing Based on The Head + The Tail

Traditional outlet

The Head

Borders 100,000 books

Blockbuster 3,000 DVDs
E-Commerce outlet

The Head + Long Tail $\quad \%$ of sales

sales from long tail

Amazon 3.7 million titles $\quad 25 \%$

Netflix 55,000 DVDs

$21 \%$

Rhapsody 1.5 million tracks $\quad 40 \%$

Wal-Mart 55,000 tracks

The concept of a long tail also describes an approach to news that can simultaneously be broader and deeper. The advantage of a long tail model for serving the public is that in addition to the head, the items in most demand - the big headlines of the day, the best sellers, the hit songs - a vast array of other items can be offered to a plethora of niche markets and idiosyncratic interests. For journalism, this approach to a daily news package can merge the front page, even the equivalent of the entire broadsheet version of the newspaper, with the Daily Me of special interests encompassing hundreds, even thousands, of topics and events. Going beyond the top news of the day to reach the vast array of niche interests of various sub-publics is important for maintaining a large audience. Reaching all these idiosyncratic sub-publics with a compelling news package is possible with a long tailmodel. It is essentially impossible with a model largely limited to the head.

Tactical Applications: The long tail not only provides a model of journalism that greatly enlarges the daily news package offered to the public; in particular, the long tail offers the opportunity for a vast expansion of a daily newspaper's local news franchise, the arena where most daily newspapers have little or no real competition.

Sports news is an excellent example that illustrates both of these points. A perennial problem for the sports editor is that sports news is not a unified kingdom with AN audience. Sports news is a rather large set of fragmented reader fiefdoms covering a

very wide array of sports, many of which - particularly the top favorites, soccer, football, basketball and baseball - are played at levels 
ranging from professional major leagues down through college and high school to organized local amateur leagues for all age groups. An online long tail is the perfect repository for this vast array of news stories, photos, and even video. Plus the long tail also can accommodate rosters, player and team photos, and a variety of statistics and other background information. Although much of this information is already available to a daily newspaper, there is no room to publish it. And for a whole new segment of the tail, detailed coverage of local amateur sports and school sports below the high school level, a substantial cadre of neighborhood volunteers can be enlisted.

The long tail model allows a news organization to make all this sports news accessible online. As Rhapsody added thousands and thousands of songs to its site, each one found a niche audience. As news organizations add more sports news items to their sites, it is likely that each one also will find a niche audience. And collectively the news audience will grow in size. Abandoning the large head/short tail model that is necessary for the print version of a newspaper in favor of a large head/long tail news package on the web will beneficially truncate the famous New York Times motto, "All the news that's fit to print" to "All the news."

Beyond sports, a news package grounded in the concept of the long tail offers the opportunity to expand the local news franchise into local neighborhoods. Again, the key advantage is the ability to serve numerous niche audiences. In the past, many large metropolitan newspapers have attempted to reach these niche audiences with zoned editions. But even with zoned editions, the amount of local news for any specific neighborhood is severely restricted by the amount of space available. And at the boundaries of the zone, the newspaper's definition of a zone or set of neighborhoods frequently did not match the perceptions and interests of residents in that area. An online long tail package of local news eliminates these problems, plus the rather onerous production and distribution problems of zoned editions in the press room and on the loading dock. Readers select whatever they want from the large set of stories available online. Nor is this kind of niche local news restricted to neighborhood audiences. There are other potential local niche audiences out there, most notably a large potential audience for local news about public education and what is happening in the schools. There is considerably more news about public education than is covered by the traditional school board/school district office beat.

Local newspapers can more fully exploit their local franchise by 
presenting more news from "the swamp," that vast expanse of public life where most concerns about public education, zoning, and other public issues are experienced by the majority of citizens. 5 The origins of many public issues that eventually reach the agenda of the school board, city council or other public institutions lie in the swamp, that messy and loosely organized conglomeration of neighborhood civic, school, religious and social associations and the casual conversations of neighbors and friends talking to each other. This is where most people participate in civic life, not at the public meetings dominated by lawyers, lobbyists, and activists.

Increasingly, these local, often ad hoc, groups have list serves and blogs that are rich sources of information about what is going on in their local setting. Monitoring these sites can identify topics of local concern - and sources for original news stories. Even a more limited role for a newspaper as an aggregator of this citizen-produced news will gain an audience and serve the information needs and desires of citizens about what is occurring in regard to these topics city-wide. There is the opportunity to tap a vast set of new sources out there in the local community. And to serve numerous niche audiences with a rich lode of information that largely has been unavailable in the past. There also are very rich opportunities here for research that identifies these niche subaudiences in our communities and that begins to explicate in detail the relevance of news from the perspective of the audience.

\section{Vertical strategy}

A news package based on the long tail model can be further elaborated and expanded through the pragmatic application to the practice of journalism of a key concept from agenda-setting theory. From the perspective of agenda-setting theory, the media agenda - agendasetting's term for what we have been calling the news package, the set of stories and information offered to the public each day - consists of two related sets of items. First, there is the agenda of topics presented in the news - the war in Iraq, the activities of a presidential candidate, a tropical storm in the Caribbean, a healthcare proposal, etc. In the abstract language of the theory, this is the agenda of objects on which the media focus attention. The term objects is used here in the same sense that social psychologists talk about attitude objects, the things that we have opinions about.

One advantage of the abstract concept, object, is that objects obviously have attributes, the variety of traits that describe and 
characterize the object. When we write a news story about an object or talk about an object, we describe it in some fashion. In other words, for each object on the media agenda, there is a corresponding agenda of attributes. But, whatever the object, the set of attributes presented in the news seldom is a full and exhaustive description of the object. Certain attributes of public figures are emphasized, others mentioned only in passing, and many not mentioned at all. The same is true for public issues. Some aspects and perspectives on these issues are emphasized, others presented only briefly, and others not mentioned at all.

In a news package limited to the large head/short tail, the news agenda must be highly selective, both in terms of which objects receive attention and which attributes of those objects are discussed. In a news package based on the long tail model, this is not necessary. Or, at least, far less necessary. In the language of agenda-setting theory, the horizontal application of the long tail model to the presentation and distribution of news gives the public the opportunity learn about a greatly expanded agenda of objects. And, the vertical application of the long tail model to the presentation and distribution of news gives the public the opportunity learn about a greatly expanded agenda of the attributes or aspects of those objects.

Tactical applications: Public issues are typically complex and encompass a variety of perspectives. And the different aspects of these issues usually are of interest to varied segments of the public. Healthcare - and the various aspects of that issue, ranging from public programs of prevention and treatment to basic research - resonate very differently among men and women, parents of young children, senior citizens, and subgroups that are especially susceptible to particular health problems. The war in Iraq has military, political, and economic aspects, among many others. Few people are interested in all of them. But many are intensely interested in one or two aspects. In short, there are numerous niche audiences for almost every public issue. Different segments of the public are interested in different aspects of an issue, and the composition of these sub-publics is constantly evolving. The internet provides the opportunity to better serve these sub-publics.

The limitations of the news hole restrict how extensively a printed daily newspaper can cover the various aspects of an issue and serve the interests of these niche sub-publics. Table 2 presents three examples of how news coverage is skewed toward particular attributes of a topic - candidates for president, the war in Iraq, and AIDS. 
Quick perusal of these examples reveals that the news stories about candidates for governor in Arizona, Colorado, Maryland, and Rhode Island published in six regional newspapers were dominated by a single attribute, strategy. This attribute accounted for more than half of the coverage. Although eight attributes of the war in Iraq were present in The New York Timescoverage during the "official war period," March 20, 2003 - May 1, 2003, two attributes account for $80 \%$ of the news even when spot news on the military conflict is set aside. And for AIDS coverage over a five-year period in five African-American newspapers, one attribute accounts for nearly half of the news and two attributes for more than $80 \%$ of the news.

Arguably, the most emphasized attributes are the most newsworthy and the most important, but experience with long tail models in ecommerce suggest that even "minor" aspects of a topic have an audience. If|we apply this model to news about public issues, we are likely to be surprised at the size of some of these niche audiences. In a time of stagnant or declining newspaper circulation, there is a need to reach and better serve as many segments of the public as possible.

To gain an audience, a news report must be relevant. And the definition of personal relevance is highly variable among the niche audiences for different public issues. Moreover, interest in the specific attributes of an issue - or any other topic in the news - is highly variable. We already know that from readership studies that track individual readers through the newspaper. Readers pick and choose among the news reports, and they vary considerably in how much of each story they read. Different aspects of the daily news resonate with different individuals. One size or color does not fit all. We know that. But in traditional news formats, we are very limited in the number of "sizes" or "colors" that can be offered. A long tail model with more topics and more attributes of those topics offers a wider variety of relevant paths through the daily news report.

Table 2 describes the patterns of news coverage for three major contemporary issues, patterns that are heavily skewed toward a small number of attributes. Applying a long tail model to the construction of news agendas on the internet offers the opportunity for a broader and more systematic presentation of public issues, election campaigns, and other news topics. Austin, Texas, attorney Nancy Lynch has advocated for some years an approach that she calls "issue mapping," the systematic presentation of the attributes of an issue, complete with a orderly catalog of the various considerations and arguments that have been made by all the parties interested in that issue. Most of this information can be 
TABLE 2. News Coverage of the Attributes of Three Public Affairs Topics

\begin{tabular}{|c|c|c|c|}
\hline \multicolumn{4}{|c|}{ A. Political Candidates * } \\
\hline \multicolumn{3}{|c|}{ Campaign strategy } & $53.9 \%$ \\
\hline \multicolumn{3}{|c|}{ Issue positions } & $31.7 \%$ \\
\hline \multicolumn{3}{|c|}{ Biography \& personal traits } & $15.2 \%$ \\
\hline \multicolumn{4}{|l|}{ B. War in Iraq ** } \\
\hline \multicolumn{2}{|c|}{ Military conflict } & $43 \%$ & \\
\hline \multicolumn{2}{|c|}{ Violence of war } & $21 \%$ & \\
\hline \multicolumn{2}{|c|}{ Human interest } & $17 \%$ & \\
\hline \multicolumn{2}{|c|}{ Anti-war protests } & $5 \%$ & \\
\hline \multicolumn{2}{|c|}{ Media coverage } & $7 \%$ & \\
\hline \multicolumn{2}{|c|}{ Responsibility } & $2 \%$ & \\
\hline \multicolumn{2}{|l|}{ Diagnostic } & $2 \%$ & \\
\hline \multicolumn{2}{|l|}{ Prognostic } & $37 \%$ & \\
\hline \multicolumn{4}{|l|}{ C. AIDS $* * * *$} \\
\hline Health & \multicolumn{2}{|c|}{$47 \%$} & \\
\hline Political & \multicolumn{2}{|c|}{$37 \%$} & \\
\hline Assets & \multicolumn{2}{|c|}{$14 \%$} & \\
\hline Moral & \multicolumn{2}{|c|}{$2 \%$} & \\
\hline
\end{tabular}

\footnotetext{
* Devitt, James. (2002).Framing gender on the campaign trail: Female gubematorial candidates and the press. Journalism \& Mass Communication Quarterly, 79(2), 445-463.

** Dimitrova, Daniela V., \& Strömbäck, Jesper. (2005). Mission accomplished? Framing of the Iraq War in the elite newspapers in Sweden and the United States. Gazette: The International Journal for Communication Studies, 67(5), 399-417.

*** Pickle, Kathryn, Quinn, Sandra Crouse, \& Brown, Jane D. (2002). HIV/AIDS coverage in black newspapers, 1991-1996: Implications for health communication and health education. Journal of Health Communication, 7, 427-444.
}

gleaned and continuously updated from news reports, blogs, and related web sites. In other words, she is urging the creation of a dynamic public affairs encyclopedia - a public affairs long tail - where any citizen can peruse as much as they want to know about the particular aspects that interest them regarding a public issue. To some degree, this could be an organized set of information from previously published news stories. But Lynch has in mind a resource that is much more systematically organized than the typical news archive and the information that can be gleaned from it by the typical search engine. But a news archive is a starting point. And taking advantage of the internet's vast capacity, one of the features becoming standard practice online is an archive of past news stories as well as copies of speeches, government and NGO reports, and other background documents related to major news stories. 


\section{Discussion}

Writing about the future of the newspaper and possible new directions in journalism has become a major cottage industry. In a new high-tech version of "names makes news," Gannett's mobile journalists, or "mojos" spend their time on the road with high-tech tools - ThinkPads, digital audio recorders, digital still and video cameras - looking for stories that are intensely local. 6 In Korea, Oh My News is an online news sources with the motto "every citizen is a reporter." About $80 \%$ of the content originates with freelance contributors, the balance from its staff of professional journalists.7 The U.S. television network, $A B C$, produces an afternoon webcast that uses video blogs, first-person essays, and other experimental ways of storytelling to present a version of the news that differs from its traditional network newscast. 8 There are many, many other examples of experimental departures from traditional practice. Although there is the capacity on the internet to entertain large numbers of these ideas simultaneously, the core appeal to an audience is relevant hard news.

A broad and deep news package grounded in the concepts of the long tail and attribute agenda can include more items that are relevant to individual members of the public. The challenge to researchers is to understand the basic dimensions of relevance that define the plethora of sub-publics that can be reached via the internet. The motivations that explain why a reader may find a news item relevant range broadly across self-interest, links to family and friends, emotional arousal, civic duty, and idiosyncratic personal appeal.9 But in the aggregate news packages tapping this variety of motivations will build large audiences.

Put another way, in more idealistic terms, this expanded agenda of news will have broader civic utility and enhance the contribution of journalism to citizenship and the functioning of a democratic society. Long ago, the Hutchins Commission on Freedom of the Press 10 outlined four key goals for the news media:

A truthful, comprehensive and intelligent account of each day's events.

Full access to the current state of affairs.

Representative picture of all social groups.

Clarification of a society's goals and values.

The internet offers the opportunity to achieve these goals in a more comprehensive way than they ever have been achieved in the past.

A civic Library of Alexandra serving the varied interests of a broad public can be built online using the concepts of the long tail and attribute 
agendas. A major component of its success will be creative studies grounded in a rich mix of research methodologies. Historian study what was. Most journalism and communication researchers study what is. But now we have a tremendous opportunity to study what could be.

\section{NOTES}

1 Roger Fidler, MediaMorphosis: Understanding New Media. Thousand Oaks, CA: Pine Forge Press, 1997. Quote on p.260.

2 The empirical research on agenda-setting, one of the key ideas that this paper draws upon, includes significant evidence that the public's use of the news media and, in turn, the influence of the news media are directly related to the perceived relevance of the news. See, for example, David Weaver's discussion of voters' need for orientation in Donald L. Shaw and Maxwell McCombs, eds., The Emergence of American Political Issues: The Agenda-Setting Function of the Press. St. Paul, MN: West, 1977. For a more recent and expanded discussion, see Jorg Matthes (2006), The need for orientation towards news media: Revising and validating a classic concept. International Journal of Public Opinion Research, 18:422-444.

In a recent Washington Post column, Frank Ahrens (Gannett To Change Its Papers' Approach. Washington Post, November 7, 2006, D01) also noted: "Gannett's ideas are shared by a growing number of people in the industry who believe that news organizations have driven away readers by becoming too imperial, too distant and not fast enough to respond to reader needs and desires."

3 Chris Anderson, The Long Tail: Why the Future of Business is Selling Less of More. New York: Hyperion, 2006.

4 Maxwell McCombs, Setting the Agenda: The Mass Media and Public Opinion. Cambridge, England: Polity, 2004.

5 David Mathews, speech to an American Press Institute seminar. Reston, VA. November 1993.

6 Frank Ahrens, A Newspaper Chain Sees Its Future, And It's Online and Hyper-Local. Washington Post, December 4, 2006; A01.

7 Oh My News. www.japanmediareview.com/japan/internet/1063672919. php 
8 Brian Stelter, $A B C$ Reshapes the Evening News for the Web. New York Times, October 12, 2007, C1 \& 7.

9 Maxwell McCombs, Personal Involvement with Issues on the Public Agenda. International Journal of Public Opinion Research, 11 (1999), 152-1.

10 Commission on Freedom of the Press, A Free and Responsible Press. University of Chicago Press, 1947.

Maxwell McCombs received his M.A. and his Ph.D. from Stanford University and his B.A. from Tulane University. McCombs holds the Jesse $\mathrm{H}$. Jones Centennial Chair in Communication at the University of Texas. McCombs is internationally recognized for his research on the agenda-setting role of mass communication. Since the original Chapel Hill study with his colleague Donald Shaw coined the term "agenda setting" in 1968, more than 400 studies of agenda setting have been conducted worldwide. 